\title{
Preliminary Framework for Human-Automation Collaboration
}

Johanna Oxstrand, Katya Le Blanc, Zachary Spielman

September 2015

The INL is a

U.S. Department of Energy

National Laboratory

operated by

Battelle Energy Alliance

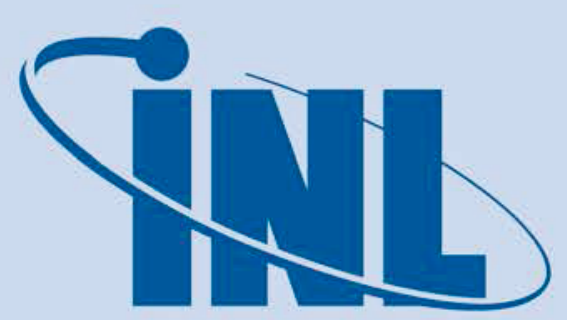

Idaho National Laboratory

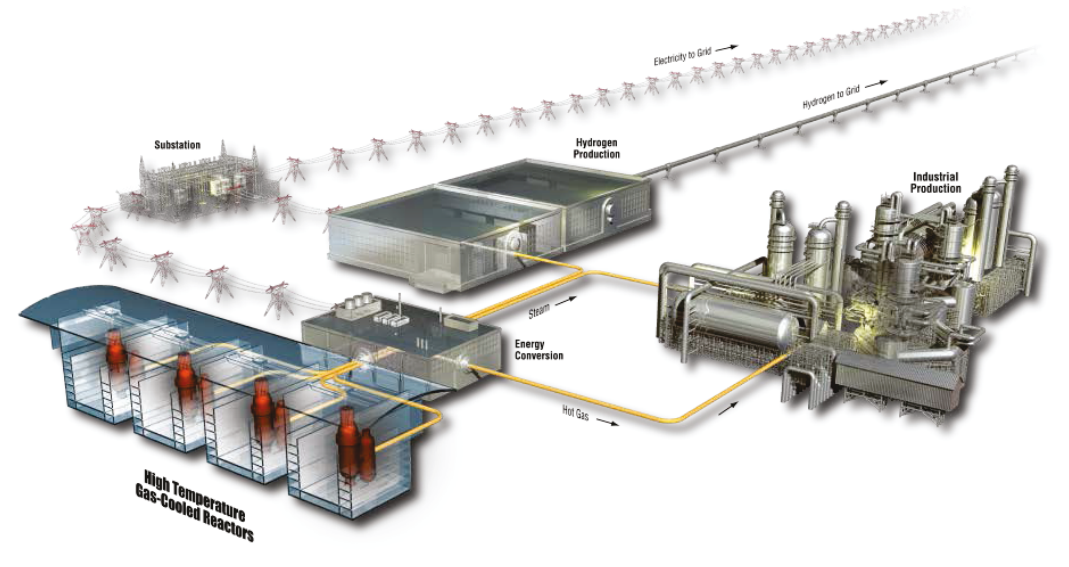




\section{DISCLAIMER}

This information was prepared as an account of work sponsored by an agency of the U.S. Government. Neither the U.S. Government nor any agency thereof, nor any of their employees, makes any warranty, expressed or implied, or assumes any legal liability or responsibility for the accuracy, completeness, or usefulness, of any information, apparatus, product, or process disclosed, or represents that its use would not infringe privately owned rights. References herein to any specific commercial product, process, or service by trade name, trade mark, manufacturer, or otherwise, does not necessarily constitute or imply its endorsement, recommendation, or favoring by the U.S. Government or any agency thereof. The views and opinions of authors expressed herein do not necessarily state or reflect those of the U.S. Government or any agency thereof. 


\title{
Preliminary Framework for Human-Automation Collaboration
}

\author{
Johanna Oxstrand, Katya Le Blanc, Zachary Spielman
}

September 2015

\begin{abstract}
Idaho National Laboratory
INL ART Program

Idaho Falls, Idaho 83415
\end{abstract}

http://www.inl.gov

Prepared for the

U.S. Department of Energy

Office of Nuclear Energy

Under DOE Idaho Operations Office

Contract DE-AC07-05ID14517 



\section{INL ART TDO Program}

\section{Preliminary Framework for Human-Automation Collaboration}

\section{INL/EXT-15-36522}

Revision 0

\section{September 2015}

\section{Author:}
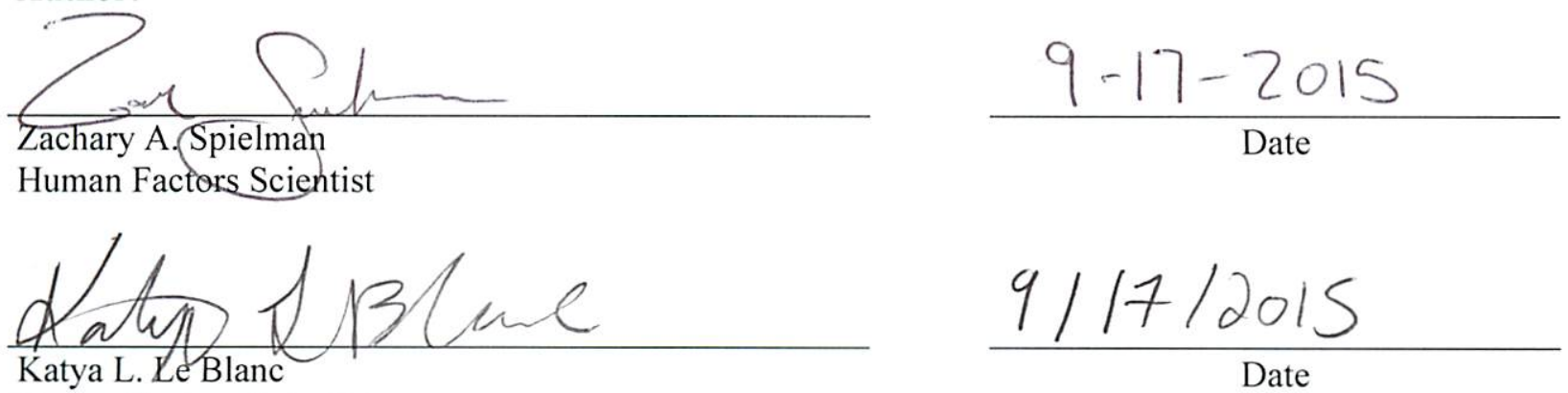

Human Factors Scientist

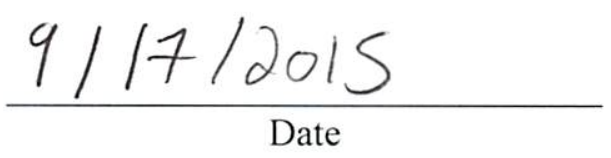

Approved by:
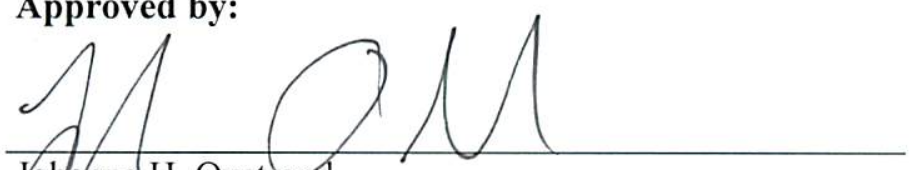

Johanna H Oxstrand

Principal Investigator

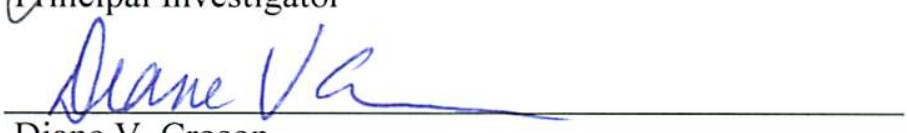

Diane V. Croson

INL ART TDO, Deputy Director
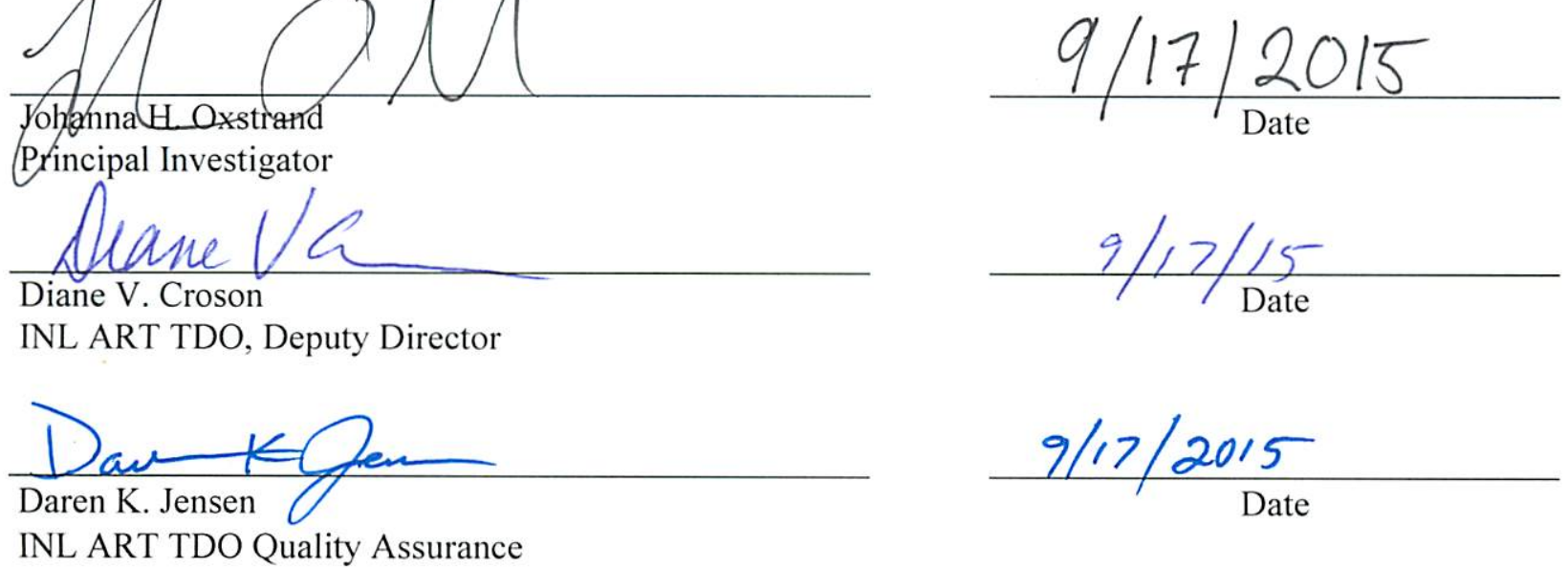

$9 / 17 / 2015$

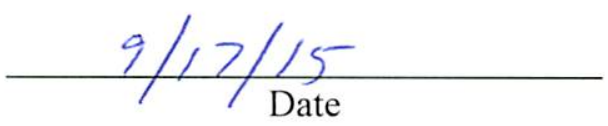

INL ART TDO Quality Assurance 


\begin{abstract}
The Human-Automation Collaboration (HAC) Research Project is sponsored by the Department of Energy's Advanced Reactor Technologies Program. The objective of the HAC Research Project is to understand how various characteristics of automation (e.g., reliability, processes, and modes) affect a nuclear power plant (NPP) operator's use and awareness of plant conditions.

As new NPP designs move towards more advanced technology and more automation, it is necessary to address concerns about how that technology and automation will affect human operator performance and the overall safety of the NPP; specifically, how the operator and automation work as a team (known as HAC) to ensure effective and safe NPP operation. The HAC Research Project research team investigated how to best design the collaboration between operators and automated systems that will have the greatest positive impact on overall NPP performance and reliability.

This report describes the development of a preliminary HAC framework, including a revision of the framework and basis for the changes made. The purpose of the framework is to addresses the methods used to analyze HAC and identify design recommendations for successful collaboration between the automation and human operator. This report also identifies additional research needed to bridge the remaining research gaps to develop a comprehensive HAC framework.
\end{abstract}




\section{CONTENTS}

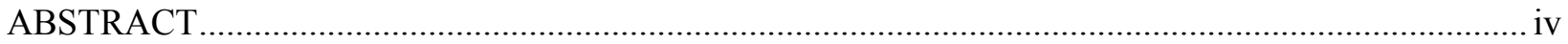

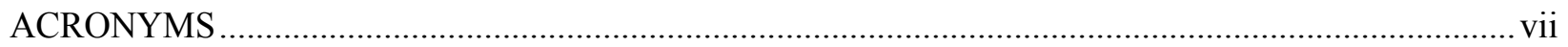

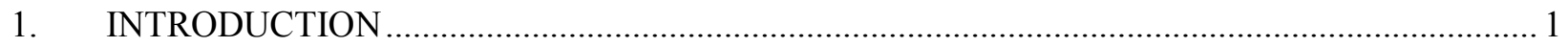

2. PRELIMINARY FRAMEWORK FOR HUMAN-AUTOMATION COLLABORATION ..............2

2.1 Phase 1-Framework for Human-Automation Collaboration .............................................. 2

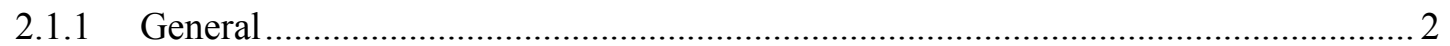

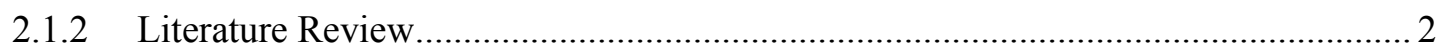

2.1.3 Development of Initial Framework ...................................................................... 3

2.2 Phase 2-Framework for Human-Automation Collaboration ........................................... 6

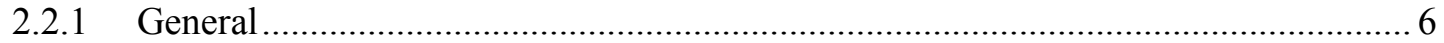

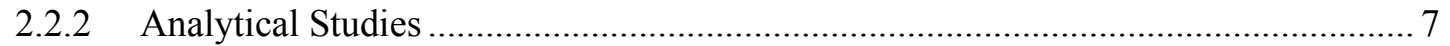

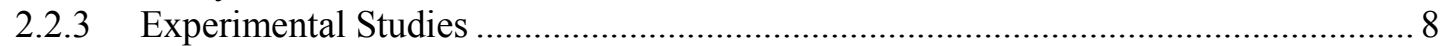

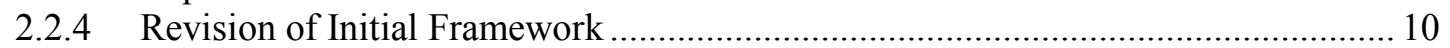

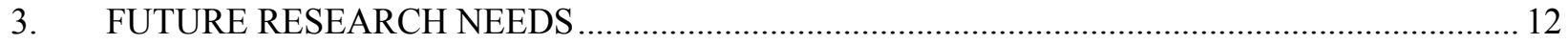

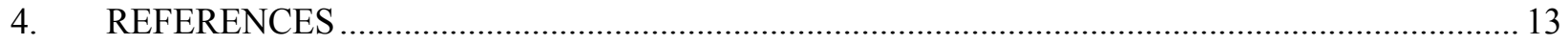

\section{FIGURES}

Figure 1. Initial preliminary framework for human-automation collaboration......................................... 2

Figure 2. Revised initial preliminary framework for human-automation collaboration. ............................ 6

Figure 3. Characterization of level of automation in revised framework. ............................................. 11

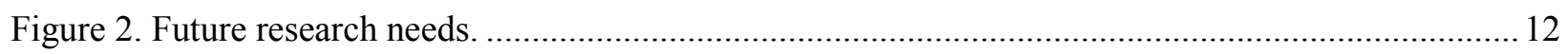

\section{TABLES}

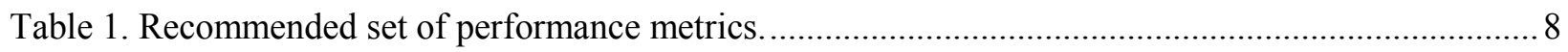




\section{ACRONYMS}

EEG electroencephalogram

HAC human-automation collaboration

HSI human-system interface

LOA level of automation

NPP nuclear power plant

SA situation awareness 


\section{Preliminary Framework for Human-Automation Collaboration}

\section{INTRODUCTION}

The Department of Energy's Advanced Reactor Technologies Program sponsors research, development and deployment activities to promote safe, technical, economical, and environmental advancements of innovative nuclear energy technologies. The focus of the human-automation collaboration (HAC) Research Project is to understand how various characteristics of automation (e.g., its reliability, processes, and modes) affect a nuclear power plant (NPP) operator's use and awareness of plant conditions.

It is expected that new NPP designs will employ technology that is significantly more advanced than the analog systems in the existing reactor fleet and use automation to a greater extent. However, moving towards more advanced technology and more automation does not necessarily imply more efficient and safer NPP operation. Therefore, concerns about how more advanced technology and automation will affect human operator performance and the overall safety of the NPP must be addressed; specifically, how the operator and automation work as a team (known as HAC) to ensure effective and safe NPP operation must be investigated. The HAC Research Project research team investigated how to best design the collaboration between operators and automated systems that will have the greatest positive impact on overall NPP performance and reliability.

The researchers conducted the investigation in two phases, which included development of a preliminary HAC framework to identify the characteristics of automation that influence how humans interact with automatic systems and how the overall human-system performs. In Phase 1, the researchers conducted a literature review and developed an initial framework to identify what factors of automation and human-automation interaction design influence performance and require a more detailed investigation in Phase 2. In Phase 2, the researchers conducted studies and further investigated factors that may influence design of a successful collaboration between the automation and human operator. Based on results of the studies and the insights gained, the researchers revised the initial framework for use in future studies. The researchers also identified additional research needs.

This report addresses development of a preliminary HAC framework, including the revision thereto and basis for changes. It addresses the methods used to analyze HAC and identifies design recommendations for successful collaboration between the automation and human operator. This report also identifies additional research needed to bridge the remaining research gaps to develop comprehensive HAC framework. 


\section{PRELIMINARY FRAMEWORK FOR HUMAN-AUTOMATION COLLABORATION}

\subsection{Phase 1-Framework for Human-Automation Collaboration}

\subsubsection{General}

In Phase 1, the researchers used the O'Hara and Higgins (2010) proposed framework to characterize six dimensions of automation as a starting point to develop a framework for HAC. The six dimensions included: 1) level of automation (LOA); 2) functions of automation; 3) processes of automation, 4) modes of automation; 5) flexibility of allocation; and 6) reliability of automation. The researchers then conducted an extensive literature review to identify the current state-of-practice of HAC. Based on the literature review, researchers selected O'Hara and Higgins (2010) proposed framework to refine and develop into an initial preliminary framework for HAC (see Figure 1). The literature review and development of the initial framework are addressed in Subsections 2.1.2 and 2.1.3.

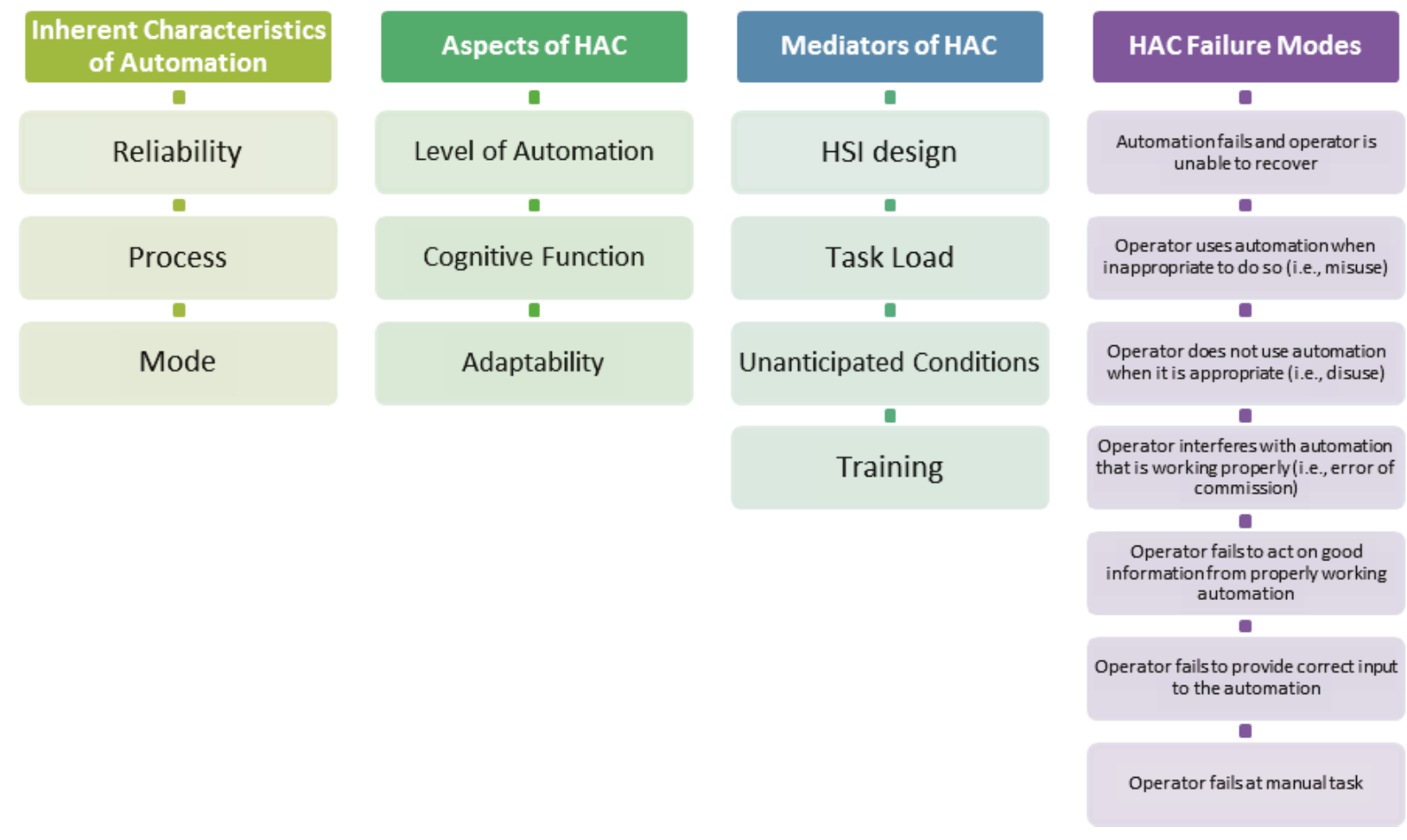

Figure 1. Initial preliminary framework for human-automation collaboration.

\subsubsection{Literature Review}

During Phase 1, the researchers conducted an extensive literature review to identify the current stateof-practice of HAC. Based on the literature review, detailed in Oxstrand et al. (2013a), the researchers refined the O'Hara and Higgins (2010) proposed framework. Development of the initial framework is addressed in Subsection 2.1.3. 


\subsubsection{Development of Initial Framework}

The findings from the literature review and the framework proposed by O'Hara and Higgins (2010) became the basis for the development of the framework for HAC. The researchers aimed to develop a framework that identifies the features of automation and human-automation interaction design that influence both human and system performance. The researchers also attempted to identify a set of features that were independent of one another (i.e., if two features appeared to overlap considerably in definition, one was removed from the list of characteristics in the framework).

To develop the initial framework, the researchers identified three main groups of characteristics associated with automation and the specific characteristics of each group. The three main groups of characteristics included inherent characteristics of automation, aspects of $\mathrm{HAC}$, and mediators of HAC.

The inherent characteristics of automation describe relative features of automation that exist independent of how HAC is designed. Some characteristics of automation that influence HAC are inherent to the automation itself. These are characteristics that need to be considered when designing HAC, but would likely be optimized with regard to automation performance rather than modified to suit a particular HAC design. The inherent characteristics of automation included in the initial framework are summarized as follows:

- Reliability. Reliability refers to how well automation accomplishes its task. Reliability is an important feature of automation due to its direct impact on system performance and effect on human performance. Generally, as reliability increases the operator's trust and use also increase, resulting in increased system performance. Unfortunately, higher reliability also tends to decrease the operator's effectiveness of monitoring the automation, leading to inappropriate actions when automation fails. This is sometimes referred to as complacency, misuse, or automation bias. While higher reliability improves task performance, it does not necessarily improve the operator's ability to detect automation failures. In fact, the higher the reliability of automation, the less likely the operator will recognize a failure. Although reliability may have a strong influence on HAC, it is reasonable to assume that designers would seek to maximize automation's reliability and address the impact on HAC through other means.

- Process. Process refers to the way an automatic system uses input from sensor feeds in the NPP and the human operator, and assesses the input relative to the automation's programmed information processing routine (e.g., control algorithms and decision logic) to initiate preprogrammed responses. When well-designed and operating correctly, the automation's preprogrammed responses are appropriate for the input it receives and assesses. However, not all automatic processes are readily comprehensible by humans. Successful HAC performance may depend on the operator knowing what the automation processes are. Although a designer may seek to make the process automation uses transparent, it is unlikely that the designer would change the process automation uses to facilitate HAC.

- Mode. Automation can be designed to have different modes, and the behavior of automation uses can be substantially different depending on the automations operation mode. One example of mode change that has potential to introduce human error is changing load following approach from turbine following mode to reactor following mode. The current mode of the system has to be clearly communicated to the operator to ensure high levels of situation awareness (SA), ensure well-informed decisions and actions, and reduce risk of diminished human performance. 
The factors identified as aspects of HAC are specific to the design of the collaboration between the automated system and human operator. These are that investigate the consequences of combining automation and human agents to control and monitor processes. Aspects of HAC include characteristics of the way HAC is designed. These factors are typically determined in the design process, more specifically, during the function allocation process. It is during this process that functions and responsibilities are assigned to the automated system, human operator, or both. The researchers focused on identifying the most influential aspects. Compared to the inherent characteristics of automation, the aspects of HAC are characteristics specific to the design of HAC rather than independent of the HAC design. The aspects of HAC included in the framework are summarized as follows:

- Level of Automation. Level of Automation (LOA) describes the amount of automation used for a particular task. The difficulty associated with LOA is to find an LOA configuration that keeps the operator's workload at an acceptable level, but also keeps the operator engaged in what is going on in the NPP. As described in Oxstrand et al. (2013a), there are several different taxonomies of LOA. While there are some differences between each taxonomy, all of them vary from fully manual (the human operator does everything) to fully automatic (the automatic system does everything), with intermediate LOAs typically involving some collaboration between the automation and human. Because it was tailored to the nuclear industry, the researchers selected the taxonomy described in O'Hara and Higgins (2010), which includes fully manual, shared control, operation by consent, operation by exception, and fully automatic for development of the HAC framework.

- Cognitive Function. The cognitive (or information processing) activities that the operator normally performs, such as detecting information, assessing information, and deciding on action, are referred to as cognitive functions in the framework. For many cognitive functions, higher LOAs produce better system performance than lower LOAs. However, for some cognitive functions, such as decision-making, higher LOAs produce poorer performance. Additionally, higher LOAs for any function tend to reduce operator monitoring and contribute to out-of-the-loop issues, which reduce the operator's ability to regain manual control after an automation failure.

- Adaptability. The LOA and cognitive function allocation between the automated system and operator can either be static by design or be designed to change dynamically. A static design implies that the LOA in the system and function allocation are permanent throughout the lifespan of the system. In the dynamic design, the LOAs and allocated functions may change depending on the circumstances. Dynamic design of automation is also described as adaptive automation. In adaptive automation, the LOAs or cognitive functions that are automated can change depending on certain criteria, or triggering conditions, such as the operator's workload or complexity of the process or evolution. Initiators can be automation initiated, operator initiated, or a combination of both (i.e., a hybrid approach). Many studies indicate that adaptive automation may be a promising way to increase the overall LOA while mitigating some of the negative consequences of high LOAs. Depending on how adaptive automation is implemented, there may be positive or negative impacts on HAC and system performance, including effects on operator SA and trust in the automation.

The mediators of HAC are contextual factors that mediate the interaction between automation and humans. There are a number of contextual factors that mediate the interaction between automation and humans. The researchers focused on identifying tangible and quantifiable factors that can be manipulated as independent variables. The researchers identified four factors and included them in the framework. Other factors that are less observable may also be relevant, such as operator trust in the automation, and future research is needed to determine how to best include such factors in the framework. More factors may be added as research progresses. The mediators of HAC included in the framework are summarized as follows: 
- Human-System Interface (HSI) Design. The design of HSI can interact with the automation design dimension to influence success of HAC. Poorly designed HSIs are implicated in many automation failures. However, in many cases, HSI can provide a means to mitigate some of the negative effects of using higher reliability and higher LOAs on human performance. For example, if HSI provides a simple means to monitor automation, the operator is more likely to do so, thus reducing monitoring problems like complacency and automation bias. Additionally, providing information about the automation (e.g., reliability, the process, and current mode) to the operator through the user interface can improve HAC and reduce the risk of the operator being out of the loop.

- Task Load. Task load is important to consider when designing HAC due to the fact that as task load increases, many of the effects of automation design dimensions increase. For example, the operator is less likely to monitor highly reliable automation under conditions of high task load. The function allocation method will allocate functions to automation, human operator, or both in a way that should optimize performance. In many cases, the function allocation also will balance workloads and achieve cost efficiencies.

- Unanticipated Conditions. When designing a system, it is impossible to predict all possible operating conditions the system will encounter. Hence, to design successful HAC, one has to consider unanticipated conditions that the operator may encounter at some point during the life of the NPP. These conditions may positively or negatively affect the ability of the automation and human to perform as expected. For example, unexpected conditions may cause the automation to fail and hence require the operator to take manual control of the system. This highlights the importance of well-designed HSI that supports a prompt and correct operator response. The unexpected conditions may also affect the operator's SA, hence impacting the operator's ability to take manual control should the automation fail.

- Training. Training can influence the extent to which the operator trusts the automation and relies or over-relies on the automation and how well the operator monitors the automation and process.

Training can have a significant impact on whether the operator is able to recognize that automation is not working properly, or take manual control when the system encounters conditions for which it was not designed to handle.

In addition to the three main groups of characteristics, the researchers identified seven failure modes that address ways in which human automation can break down. These failure modes were the product of a combination of unfavorable automation characteristics, HAC aspects, and/or mediating variables. The researchers included the HAC failure modes as a separate framework group. The HAC failure modes included in the framework are as follows:

- Automation fails and operator is unable to recover

- Operator uses automation when inappropriate to do so (i.e., misuse)

- Operator does not use automation when it is appropriate (i.e., disuse)

- Operator interferes with automation that is working properly (i.e., error of commission)

- Operator fails to act on good information from properly working automation

- Operator fails to provide correct input to the automation

- Operator fails at manual task. 


\subsection{Phase 2-Framework for Human-Automation Collaboration \\ 2.2.1 General}

In Phase 2, the researchers conducted follow-on activities using the initial preliminary framework for HAC developed during Phase 1 as the foundation. The follow-on activities were tailored to investigate specific research gaps identified by the literature review and allowed further investigation of factors that may influence the design of a successful collaboration between the automation and human operator. The follow-on activities included a series of in-depth analytical studies and experimental studies.

Based on results of the analytical and experimental studies, the researchers revised the initial preliminary framework for HAC developed during Phase 1 to improve the framework (see Figure 2). The analytical and experimental studies and resulting revision of the initial framework are addressed in Subsections 2.2.2, 2.2.3, and 2.2.4. Finally, the researchers identified additional research needed to bridge the remaining gaps before comprehensive framework for HAC can be developed. These research needs are addressed in Section 3.

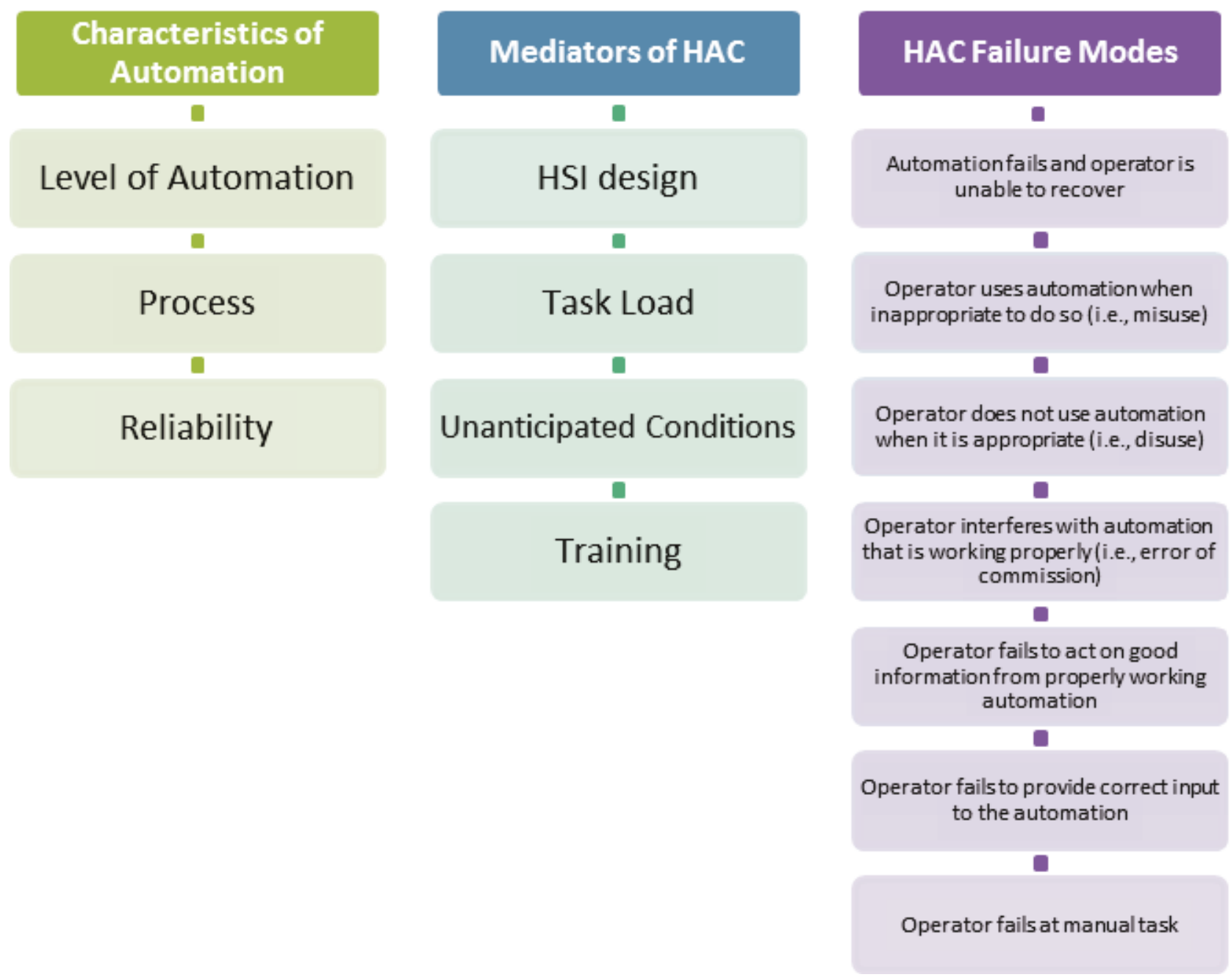

Figure 2. Revised initial preliminary framework for human-automation collaboration. 


\subsubsection{Analytical Studies}

During Phase 2, the researchers first conducted three in-depth analytical studies to explore the effects on teamwork when the automated system is viewed as a part of the team, identify performance measures specific to HAC in the nuclear domain, and investigate triggering conditions for adaptive automation. Summarized descriptions and results of the analytical studies are provided in the following subsections. Detailed descriptions of the methodology, results, and conclusions for the studies are provided in Oxstrand et al. (2013b).

2.2.2.1 Models of Teamwork. The teamwork analytical study focused on identifying the requirements for effective human-automation teamwork that are appropriate in a commercial NPP team environment. Once identified, the researchers elaborated on the specific requirements of automation and human team members, which can be used to develop guidance for what automation characteristics are needed to be good "team players."

The researchers sought to identify what makes an effective team-in particular, what makes an effective team in the nuclear domain. To do so, the researchers evaluated different models of human-human teamwork. As a part of the evaluation, the researchers reviewed recent research on models of human teamwork; focused on NPPs and other complex, highly-automated systems; and identified general principles about what makes an effective team and the characteristics needed.

The teamwork analytical study suggested that the demands on human-automation interaction might be the greatest when the automation agent is supporting higher-level cognitive process, such as situation assessment and response planning. Results of the teamwork study suggested the following requirements for designing human-automation interaction:

- The way information is processed should be accessible to the operator

- The design of communication functions and features should enable the operator to obtain the information needed to use automation information, such as assessing credibility of the results

- Interruptions should be minimized

- The level of detail should be controlled

- The operator should be able to provide input to the processing and direct its activities.

\subsubsection{Standardized Human-Automation Collaboration Performance Measurement}

Battery. To accomplish the goal of facilitating optimal operator and NPP performance in a highly-automated system, it was necessary to measure performance of the system, operator, NPP, and human-automation interaction in the specific NPP context. The performance measurement battery analytical study aimed to develop an initial set of performance measures that were applicable in the nuclear domain and focus on the relationship between automation and humans, including operator awareness, trust in the automation, and use of automation, as well as measures that reflect multi-agent teamwork.

The researchers concluded that objective performance measures were the most direct measures of human and system performance. However, these measures should be used in conjunction with subjective measures to validate the objective measures and better detect ceiling effects. The subjective measures can also be used to gain qualitative insights useful for future system design. The researchers recommended use of the set of metrics described in Table 1 to measure primary task performance, SA, workload, trust, and system performance. 
Table 1. Recommended set of performance metrics.

\begin{tabular}{|l|l|l|}
\hline \multicolumn{1}{|c|}{ Measure } & \multicolumn{1}{|c|}{ Recommended Metric(s) } & \multicolumn{1}{c|}{ Comments } \\
\hline $\begin{array}{l}\text { Primary task } \\
\text { performance }\end{array}$ & $\begin{array}{l}\text { - Accuracy (or errors) } \\
\text { - Time to complete } \\
\text { - Time to initiate }\end{array}$ & $\begin{array}{l}\text { Expert observation should be used in earlier stages of research } \\
\text { and more objective measures should be used in experiments and } \\
\text { validation of designs. }\end{array}$ \\
\hline SA & $\begin{array}{l}\text { - SA control room inventory } \\
\text { - Eye gaze }\end{array}$ & $\begin{array}{l}\text { SA control room inventory is the preferred method; however, eye } \\
\text { tracking can also be used when it is practical. }\end{array}$ \\
\hline Workload & $\begin{array}{l}\text { National Aeronautics and Space } \\
\text { Administration task load index }\end{array}$ & \\
\hline Trust & Jian et al. (2000) trust scale & $\begin{array}{l}\text { A trust measure should be developed specifically for } \\
\text { nuclear-specific HAC research. However, the Jian et al. (2000) } \\
\text { trust scale is sufficient until a different method is developed. }\end{array}$ \\
\hline $\begin{array}{l}\text { System } \\
\text { performance }\end{array}$ & $\begin{array}{l}\text { - Function performance } \\
\text { Discrepancy scores }\end{array}$ & $\begin{array}{l}\text { Both metrics should be used together and results compared with } \\
\text { objective and subjective measures of human performance. }\end{array}$ \\
\hline
\end{tabular}

2.2.2.3 Initiators and Triggering Conditions for Adaptive Automation. The purpose of the study of initiators and triggering conditions for adaptive automation was to identify the triggering mechanisms that provided the best support for operator and system performance, and were likely to be achievable in the NPP control room context.

A review of adaptive automation literature indicated that there were tradeoffs associated with each triggering mechanism, but that some were more applicable to the nuclear domain. The two mechanisms that consistently improve performance in laboratory studies were operator-initiated adaptive automation based on hierarchical task delegation and the electroencephalogram (EEG)-based measure of engagement. Current EEG methods are intrusive and require extensive analysis; therefore, the EEG-based measure of engagement is not recommended for control rooms at this time.

In addition, the researchers studied both adaptive and adaptable automation (i.e., when the shift of LOAs is controlled by triggering conditions [adaptive] and when the shift in automation is controlled by the operator [adaptable]). The researchers concluded that adaptable automation using a task delegation interface may be the best way to approach automation for new NPPs due to its tendencies to enhance performance compared to static automation. In this scheme, the automation can be shifted up and down the abstraction hierarchy based on the current conditions or operator's needs.

\subsubsection{Experimental Studies}

The follow-on activities leading up to the experimental studies indicated that using intermediate LOAs was ideal for keeping operators engaged and that intermediate LOAs were ideal for performance. Due to the assumption that new NPPs will be highly automated, it is important to investigate how to enable higher LOAs, while still keeping the operator actively engaged in operation of the NPP.

The researchers concluded that literature supports the claims that adaptable automation may be effective in enhancing performance compared to fully manual and that SA may be better when using adaptable automation compared with using fully automated systems. The overall promise of adaptive (and adaptable) automation is that it provides system performance similar to fully automated designs, but without the negative human performance consequences. However, there are gaps in the literature. For example, few studies actually compared adaptive and adaptable automation to intermediate LOAs; instead, most studies focused on comparing the extremes (i.e., fully manual and fully automatic). Therefore, the researchers decided to investigate whether adaptable automation manages human performance and system performance tradeoffs more effectively than static intermediate LOAs. 
To further explore the impact of adaptable automation compared to intermediate LOAs, the researchers conducted two experimental studies. In addition to adaptable automation, the studies also investigated participants' SA and workloads during different automation conditions. Summarized descriptions and results of the experimental studies are provided in the following subsections. Detailed descriptions of the studies are provided in Oxstrand and Le Blanc (2014) and Le Blanc et al. (2015).

2.2.3.1 First Experimental Study. A simplified process control simulation was developed to enable the researchers to investigate the interaction and collaboration between the human participants and system in a controlled manner. The process simulation was also developed to be generalized to the context of NPPs. The process was designed to be simple enough to allow undergraduate psychology students to operate with minimal training, but complex enough to allow for varying the LOA. The process also needed to be difficult enough for researchers to detect differences in performance across the automation conditions. The process required the management of two separate product streams with different operational requirements. One of these product streams had dynamic requirements. This design mimicked integrated energy systems and load-following, which are potential operational concepts for small modular reactors.

The process simulation was designed with the following four possible LOAs:

1. Fully Manual. Operator handles all tasks manually.

2. Intermediate. Automation monitors, generates responses, and presents possible actions to the operator and the operator chooses the actions to be taken; automation then carries out the chosen action.

3. Fully Automatic. Automation handles all tasks; operator's task is to monitor and take manual control if necessary.

4. Adaptable. With the process divided into four high-level tasks, operator can choose to delegate any (or all) of the four tasks to the automation or manually perform the tasks.

The main independent variable was the automation condition, which was manipulated between participants (each participant executed both scenarios using a single LOA). The other independent variable was the scenario type (introduced automation fault and no fault). This was manipulated within participants where each participant did one of each scenario type. The automation fault was the same for each participant. During the fault scenario, one pump failed to turn on automatically when a valve was opened. The participant had to manually control the pump to recover from the fault and keep the parameter in range. The participants were trained on how to do so before starting the scenario. The fault was injected at the same time for each participant.

The researchers applied results of the performance measure battery analytical study described in Subsection 2.2.2.2 to design the specific metrics for the study. The researchers measured system performance by assessing the degree to which the parameters were kept within range. In addition, the researchers measured the total time that any one parameter was out of range. SA and workload were also measured.

The researchers concluded that results from the experimental study do not confirm the common claim that adaptable automation is an effective method to manage human performance and system performance tradeoffs associated with increasing automation, but do not necessarily refute it. Results indicated that performance using adaptable automation is similar to that of using intermediate LOAs. This does not support the claim that adaptable automation is a better solution than intermediate LOAs, which is often stated by other researchers. 
However, it is also important to note that there were other results from the study that were not consistent with existing literature. These inconsistencies could have been due to some of the limitations present in the experimental study. Some of the identified limitations included the following:

- The study was conducted with university students who received minimal training

- The process control simulation was not sufficiently complex to design a truly hierarchical abstraction scheme needed to detect differences between adaptable automation and intermediate LOAs

- The definition for an intermediate LOA used in the study does not represent static automation.

2.2.3.2 Second Experimental Study. The second experimental study aimed to investigate the effects that automation has on overall human-system performance. As in the first study, the researchers employed a simplified process control simulation using a nested experimental design to evaluate performance differences between four LOAs (manual, intermediate, adaptable, and automatic) with varying faults and operational condition changes occurring during each scenario. The effects of automation were measured by participants' abilities to detect and respond to failing automation and conditional changes using a variety of metrics assessing SA, system performance, and workload.

Results of the second study indicated that more automation leads to better system performance under normal operating conditions, and poorer SA and system performance under fault conditions. Results for the two middle LOAs were not as straightforward. Consistent with the hypothesis, system performance using adaptable automation exceeded system performance when using the intermediate LOA. However, contrary to the hypotheses, SA and fault performance using the adaptable LOA were not superior to SA and fault performance using the intermediate LOA. Results of the second study indicated that, although adaptable automation fulfills half if its promise (i.e., it produces system performance closer to fully automatic than intermediate LOAs), it falls short of also enhancing SA and fault performance. The second study demonstrated the fundamental tradeoff associated with high LOAs: automation enhances performance during normal conditions, but increases failure (sometimes catastrophically) of the human system under the condition of automation failure. In contrast, although manual performance is inferior to automatic performance under normal conditions, it is more stable and superior to automatic performance under automation failure conditions. An intermediate LOA provided similarly good fault management performance, but did not enhance system performance as well as an adaptable LOA under normal conditions did.

Unfortunately, similar to the first study, the second study did not demonstrate that an adaptable LOA was an effective approach to avoiding that fundamental tradeoff and achieving an optimal combination of system performance, SA, and fault management performance. Further, research is needed to identify an approach to HAC that will enable higher LOAs without succumbing to the fundamental tradeoffs of automation.

\subsubsection{Revision of Initial Framework}

Based on results from the analytical and experimental studies, the researchers modified the initial framework. The experimental studies revealed that many of the characteristics identified in the initial framework seemed independent when presented in abstract theoretical context, but overlapped considerably when applied to an actual system. For example, LOA, cognitive function of automation, and adaptability overlap so much that the influence on human performance cannot be separated. The majority of taxonomies of LOA combine the amount of automation (defined as LOA in the initial framework) and the kind of information processing function that is automated (defined as cognitive function of automation in the initial framework). The researchers discovered that generally, as more is automated, more cognitive functions are also automated. Therefore, in the Phase 2 framework, LOA and cognitive function were combined and referred to as LOA. The experimental studies also revealed that the level of adaptability could be defined in the LOA. For example, only the endpoints on the LOA scale are static (fully manual and fully automatic). LOAs on the middle of the scale are by definition dynamic. The degree of how 
dynamic they are can vary, but is not fully independent of the LOA.

The researchers proposed a characterization of LOA that reflected the relationship between how much was automated, what kinds of information processing activities were automated and how dynamic the allocation between the human and automation was (Figure 3).

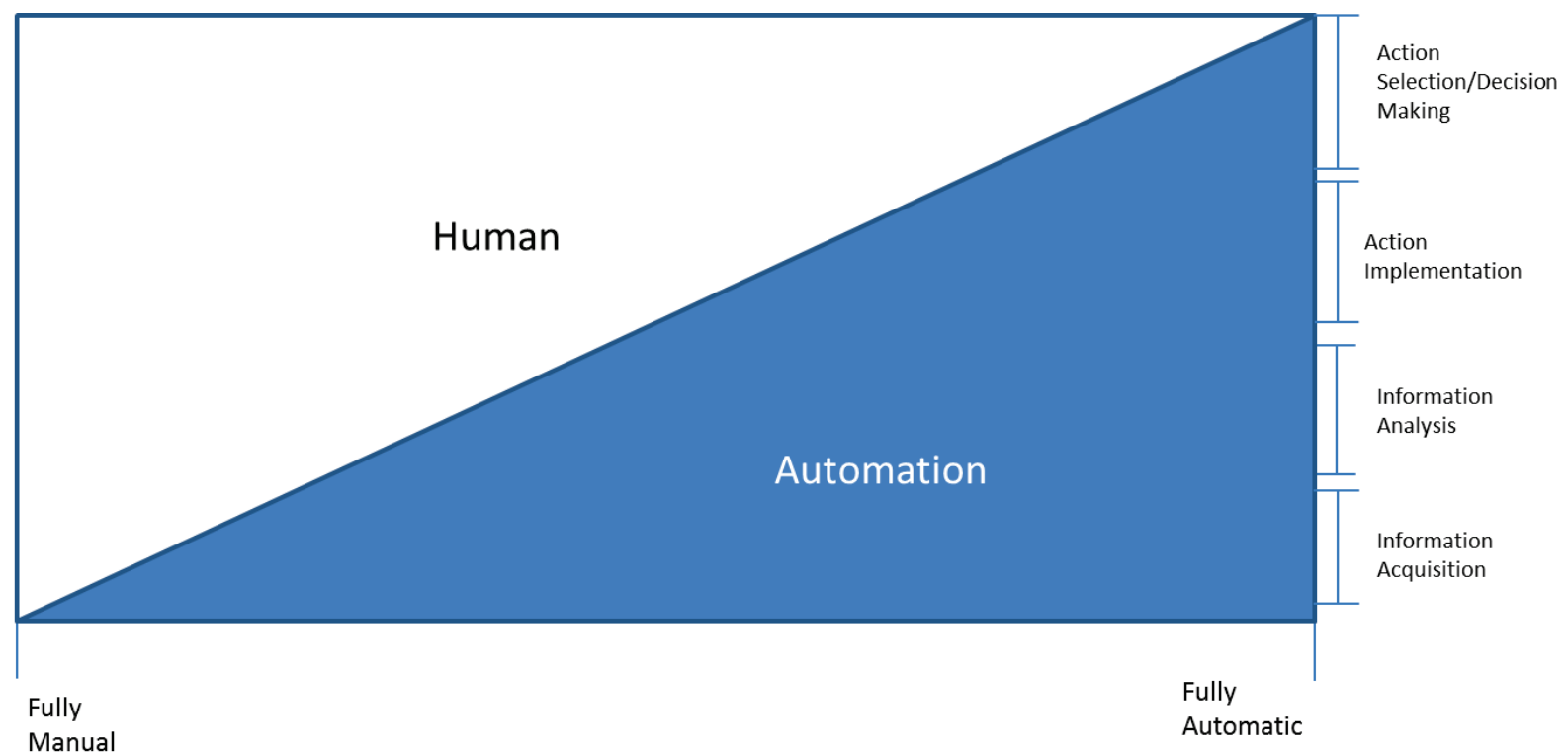

Figure 3. Characterization of level of automation in revised framework.

In Figure 3, the $x$ axis represents the level of control authority delegated to each agent (human or automation). The $y$ axis represents the stage of human information processing that is carried out by each agent. For example, a decision support system might monitor NPP data, make calculations, and compare data to specified ranges, and then suggest a course of action to the human operator. The operator can take the suggested course of action and the automation can implement it, or the operator can decide to implement an alternative (and carry it out manually). Note that decision support in this case is inherently dynamic, as the control authority shifts up and down the continuum depending on the situation. This model depicts who has authority, what stage of information processing is executed by each agent, and whether the allocation is dynamic. This characterization of LOA enabled three related concepts (LOA, cognitive function, and adaptability) to be more accurately represented as a single construct with two dimensions. Therefore, it was represented as a single construct in the revised framework.

Mode of automation also overlapped with other characteristics in the initial framework. Mode of automation is related to both the LOA (some automation modes simply change the LOA) and the process' automation use (some modes of automation change the way the automation accomplishes its task). Therefore, mode of automation was removed from the revised framework. 


\section{FUTURE RESEARCH NEEDS}

Additional research needs were identified as a result of the conducted research in the HAC Research Project. As illustrated in Figure 2, these needs are summarized in two main groups: additional experimental studies and the development of a HAC evaluation tool. Phase 2 experimental studies concluded that adaptable LOAs might not be superior to intermediate LOAs, nor an effective way to avoid tradeoffs associated with high LOAs. These results are interesting since they contradict findings made by Clamann et al. (2002), Kaber and Endsley (1997), and Parasuraman et al. (2009). The contradicting results may be due to simplified process simulations and the use of participants naïve to nuclear operations. Therefore, it is important to increase the validity and extend the research scope by conducting a series of experiments in a realistic setting using experienced NPP operators.

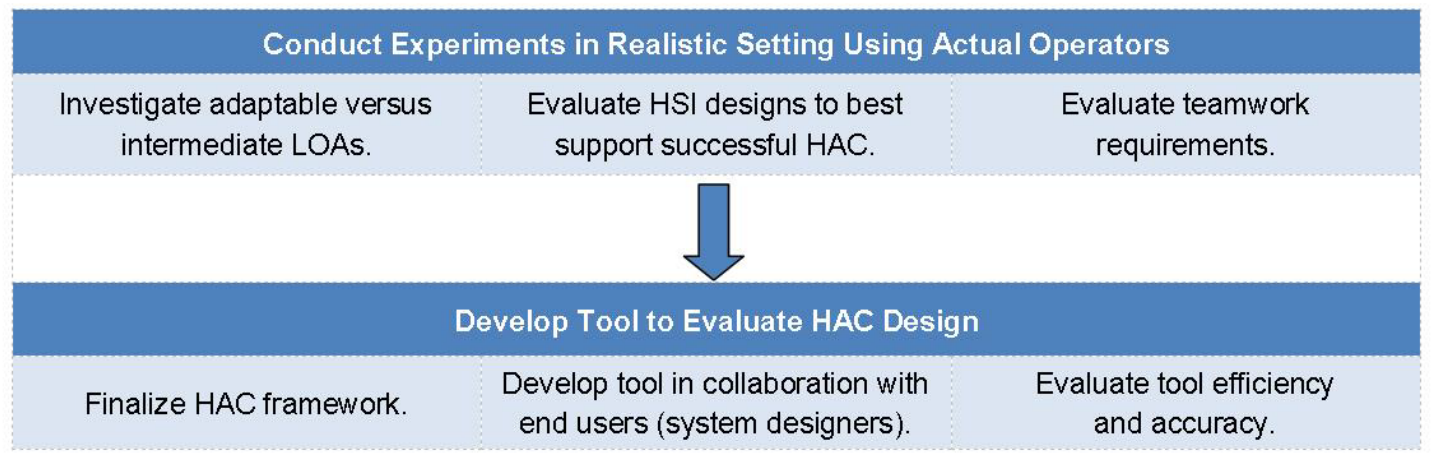

Figure 2. Future research needs.

Future experiments should further investigate the relationship between adaptable LOAs and intermediate LOAs to determine the generalizability of the results from the two HAC experimental studies to the nuclear industry. The additional series of experiments should focus on how to design HSI to best support successful collaboration between the system and operator. These experiments are contingent on results from further investigation of adaptable versus intermediate LOAs since these two approaches pose different requirements on the HSI design. A parallel activity should be used to evaluate the teamwork requirements that the HAC researchers identified. The focus of these experiments should be to identify which requirements have the largest impact on the overall HAC design and decide if and how those requirements should be incorporated into the HAC framework.

The second main research need to address in the future is the development of an evaluation tool to inform HAC design under various contexts. The purpose of the tool would be to ensure that integrated human-automation system performance acceptably meets design performance requirements for both production and safety. The first step toward developing such a tool would be to finalize the HAC framework based on the findings from the series of experimental studies. When the framework is complete, it should be converted into a tool to support system designers' evaluations of different HAC design solutions. The end users (the system designers) should be involved in the development process to ensure their needs are identified and met. Before the tool is launched, it must be evaluated (and potentially revised) to ensure it adequately meets both the requirements posed by the HAC framework and users' needs. 


\section{REFERENCES}

Clamann, M.P., M.C. Wright, and D.B. Kaber, 2002, "Comparison of performance effects of adaptive automation applied to various stages of human-machine system information processing," in Proceedings of the Human Factors and Ergonomics Society Annual Meeting, Volume 46, Issue 3, pp. 342-346.

Jian, J., A.M. Bisants, and C.G. Drury, 2000, "Foundations for an empirically determined scale of trust in automated systems," International Journal of Cognitive Ergonomics, Volume 4, Issue 1, pp. 53-71.

Kaber, D.B., and M.R. Endsley, 1997, "Out-of-the-loop performance problems and the use of intermediate levels of automation for improved control system functioning and safety," Process Safety Progress, Volume 16, Issue 3, pp. 126-131.

Le Blanc, K., Z. Spielman, G. Bower, and J. Oxstrand, 2015, Enabling Situation Awareness Under High Levels of Automation: Results From An Experimental Study, INL/EXT-15-35791, Rev. 0, Idaho National Laboratory.

O'Hara, J., and J. Higgins, 2010, Human System Interfaces to Automatic Systems: Review Guidance and Technical Basis, BNL Technical Report 91017 2010, Upton, NY: Brookhaven National Laboratory.

Oxstrand, J., and K. Le Blanc, 2014, Effects of Levels of Automation for Advanced Small Modular Reactors: Impacts on Performance, Workload, and Situation Awareness, INL/EXT-14-32639, Rev. 0, Idaho National Laboratory.

Oxstrand, J., J. O'Hara, K.L. Le Blanc, A.M. Whaley, J.C. Joe, and H. Medema, 2013a, Development of an Initial Model of Human-Automation Collaboration - Results from a Needs Analysis, INL/EXT-13-28682, Rev. 1, Idaho National Laboratory.

Oxstrand, J., K.L. Le Blanc, J.C. Joe, A.M. Whaley, H. Medema, and J. O’Hara, 2013b, Framework for Human-Automation Collaboration: Conclusions from Four Studies, INL/EXT-13-30570, Rev. 0, Idaho National Laboratory.

Parasuraman, R., K.A. Cosenzo, and E. De Visser, 2009, “Adaptive Automation for Human Supervision of Multiple Uninhabited Vehicles: Effects on Change Detection, Situation Awareness, and Mental Workload," Military Psychology, Volume 21, Issue 2, pp. 270-297. 